\title{
Hidden variable theories and quantum nonlocality
}

\author{
A D Boozer \\ Department of Physics, California Institute of Technology, Pasadena, CA 91125, USA \\ E-mail: boozer@caltech.edu
}

Received 28 October 2008, in final form 22 December 2008

Published 9 February 2009

Online at stacks.iop.org/EJP/30/355

\begin{abstract}
We clarify the meaning of Bell's theorem and its implications for the construction of hidden variable theories by considering an example system consisting of two entangled spin-1/2 particles. Using this example, we present a simplified version of Bell's theorem and describe several hidden variable theories that agree with the predictions of quantum mechanics. These example theories clarify some subtle points, which are often misunderstood, regarding what it is that Bell's theorem actually establishes.
\end{abstract}

\section{Introduction}

Ever since quantum mechanics was first developed, people have been troubled by the fact that it is indeterminate: given the quantum state of a system, we cannot predict with certainty the outcomes of measurements performed on the system. In a 1935 paper, Einstein, Podolsky and Rosen (EPR) argued that this indeterminacy shows that quantum mechanics is necessarily incomplete, and that beneath quantum mechanics must lie a more fundamental theory in which measurement outcomes are determined by hidden variables that quantum mechanics does not account for [1]. A key premise of the EPR argument is the assumption of locality, that is, the assumption that influences cannot propagate faster than the speed of light. This assumption seemed above reproach until 1964, when John Bell refuted the EPR argument by showing that the locality assumption must fail for any theory that agrees with the predictions of quantum mechanics [2]. Bell's insight revealed a deep and previously unexpected property of the world, and has profound implications for the EPR programme of replacing quantum mechanics with a hidden variable theory ${ }^{1}$.

In this paper, we present a simplified version of Bell's theorem, and we describe several example hidden variable theories. These example theories clarify some subtle points, which are often misunderstood, regarding what it is that Bell's theorem actually establishes. The

1 An overview of the EPR argument and Bell's theorem that is accessible to undergraduates is given in [3], and an experiment that demonstrates quantum nonlocality in an undergraduate laboratory is discussed in [4]. 
arguments involved are mathematically simple and require little background in physics, but illustrate profound features of the world and help clarify what it is that is strange and novel about quantum mechanics. In addition, the arguments rely in a critical way on the properties of entangled states, which are an area of active research and play a key role in fields of current interest such as quantum computation and quantum information science. The paper should be accessible to undergraduates, and is intended to supplement an introductory quantum mechanics class.

The paper is organized as follows. In section 2, we introduce the concept of a hidden variable theory. In section 3 we describe a simplified version of the EPR argument, which attempts to show that quantum mechanics is necessarily incomplete and must ultimately be replaced by a hidden variable theory. In section 4 , we describe a model system consisting of two entangled spin-1/2 particles, derive the quantum-mechanical predictions for the behaviour of the system and discuss the possibility of explaining this behaviour in terms of a hidden variable theory. In section 5 we present a simplified version of Bell's theorem, which shows that there are important restrictions on the types of hidden variable theories that can explain the behaviour of the model system. In sections $6-8$, we describe three example hidden variable theories that are allowed by Bell's theorem.

\section{Hidden variable theories}

Consider a physical system, such as a free particle, and imagine that we perform a measurement on the system to determine the value of a physical quantity $P$, such as the particle's position or angular momentum. According to a common-sense view of the world, the value of $P$ is present in the system prior to the measurement, and the measurement process simply reveals this preexisting value to us. According to the standard interpretation of quantum mechanics, however, this common-sense view sometimes fails: in certain cases, the value of $P$ is not present in the system prior to the measurement; instead, it is determined in part by the measurement process itself.

As an example, let us consider the quantum description of a spin-1/2 particle. The state of the particle is given by a spinor $|\hat{n}\rangle$, and if we measure the spin of the particle along the $\hat{r}$-axis the probabilities of obtaining values $\pm 1 / 2$ are

$$
p_{ \pm}=(1 / 2)(1 \pm \hat{r} \cdot \hat{n})
$$

Suppose that the particle is in the state $|\hat{z}\rangle$. If we measure $S_{z}$, the spin of the particle along the $\hat{z}$-axis, we can predict with certainty that we will obtain the result $+1 / 2$, so in this case the common-sense view applies. However, if we measure $S_{x}$, all we can say is that there are equal chances of obtaining $+1 / 2$ and $-1 / 2$. So in this case, the common-sense view fails: the quantity $S_{x}$ does not have a definite value before the measurement is performed.

Because of this kind of indeterminacy, EPR thought that quantum mechanics gives only a partial description of the world, and that beneath quantum mechanics must lie another theory to which the common-sense view applies. This underlying theory would show that measurement outcomes are actually determined by hidden variables that quantum mechanics does not account for. According to such a theory, when we prepare an ensemble of systems, each in the same quantum state, different systems in the ensemble are assigned different values of the hidden variables, and the probability distribution of measurement outcomes is determined by the distribution of hidden variables across the members of the ensemble. Thus, the indeterminacy of the measurement outcomes is attributed to ordinary statistical uncertainty rather than to a fundamental indeterminacy in the objective world. 
To illustrate this idea, let us return to the example of a spin- $1 / 2$ particle and show how we can account for the probabilities given in equation (1) by a hidden variable theory ${ }^{2}$. In this hidden variable theory the quantum state $|\hat{n}\rangle$ is associated with an ensemble of systems, each of which is assigned a randomly chosen unit vector $\hat{m}$. Thus, the state of any given system can be characterized by the pair of unit vectors $(\hat{n}, \hat{m})$. We stipulate that for the ensemble associated with state $|\hat{n}\rangle$ the distribution of unit vectors $\hat{m}$ is uniform over the surface of the sphere, and that for a system in state $(\hat{n}, \hat{m})$ the value of a spin measurement along the $\hat{r}$-axis is

$$
v(\hat{n}, \hat{m})= \begin{cases}+1 / 2 & \text { if } \quad(\hat{n}+\hat{m}) \cdot \hat{r}>0, \\ -1 / 2 & \text { if } \quad(\hat{n}+\hat{m}) \cdot \hat{r}<0 .\end{cases}
$$

Note that for any particular system, we can predict with certainty the outcome of a spin measurement along any axis. However, because an ensemble is made up of systems with different values of $\hat{m}$, for the ensemble as a whole we can only say that certain measurement outcomes occur with certain probabilities. Using equation (2), it is straightforward to show that these probabilities agree with the probabilities predicted by quantum mechanics in equation (1).

\section{The EPR argument}

We have shown how a hidden variable theory can be constructed for the specific case of a spin$1 / 2$ particle, but one could imagine that a more general hidden variable theory could account for all physical systems. If such a hidden variable theory really did describe the world, it would mean that quantum mechanics is incomplete and gives only a partial description of the world. This is an appealing possibility, because it would allow us to salvage the common-sense view in which measurement outcomes reflect values that are present in the system before the measurements are performed. But EPR went further than discussing the mere possibility that quantum mechanics might be incomplete: they presented an argument that was intended to show that quantum mechanics is necessarily incomplete.

We describe here a simplified version of the EPR argument due to Bohm [7]. Consider two spatially separated spin- $1 / 2$ particles in the quantum state ${ }^{3}$

$$
|\psi\rangle=(1 / \sqrt{2})(|\hat{z}\rangle \otimes|-\hat{z}\rangle-|-\hat{z}\rangle \otimes|\hat{z}\rangle)
$$

Suppose that we measure the spin of the first particle along the $\hat{z}$-axis and then measure the spin of the second particle along the $\hat{z}$-axis. We will assume that the two measurement events are space-like separated, so influences moving slower than the speed of light cannot propagate from the first measurement event to the second. According to quantum mechanics, before the measurements are performed neither particle has a definite value of $S_{z}$ and thus we cannot predict the measurement outcomes. But after the first measurement is performed the state vector collapses to either $|\hat{z}\rangle \otimes|-\hat{z}\rangle$ or $|-\hat{z}\rangle \otimes|\hat{z}\rangle$, and the particles now have definite values of $S_{z}$. Thus, once we know the outcome of the first measurement we can predict with certainty the outcome of the second measurement. At this point, EPR make the locality assumption: because the two measurement events are space-like separated, they assume that the measurement performed on the first particle could not have changed the spin value of the second particle. Given the locality assumption, together with the fact that the second particle has a definite value of $S_{z}$ after the first measurement has been performed, EPR conclude that the second particle had a definite value of $S_{z}$ even before the first measurement was

2 This example is discussed in [5] and is based on a similar example given in [6].

3 Note that $|\psi\rangle$ is an entangled state, that is, it cannot be written in the form $|\psi\rangle=|\alpha\rangle \otimes|\beta\rangle$ for any states $|\alpha\rangle,|\beta\rangle$. 
performed. But quantum mechanics was incapable of telling us what this value was; thus, quantum mechanics must be incomplete.

\section{The model system}

In 1964, John Bell refuted the EPR argument by showing that the locality assumption must fail for any theory that agrees with the predictions of quantum mechanics [2]. In section 5 we will present a simplified version of Bell's argument, but before doing so let us consider a model system that is based on the entangled spin- $1 / 2$ particles that we considered above. Let us assign each particle a detector that can be set to measure the particle's spin along one of three axes $\hat{e}_{0}, \hat{e}_{1}, \hat{e}_{2}$, where

$$
\hat{e}_{n}=\cos (2 \pi n / 3) \hat{x}+\sin (2 \pi n / 3) \hat{y} \text {. }
$$

We will let $A$ and $B$ denote the detectors corresponding to the first and second particles, and we will let $A i$ and $B i$ denote the situation in which detectors $A$ and $B$ are set to measure the spin along axis $\hat{e}_{i}$. Also, we will let $a x$ and $b x$ denote the situation in which detectors $A$ and $B$ register outcome $x$, where $x$ can be be either $u=+1 / 2$ or $d=-1 / 2$.

Imagine that we perform a series of experiments on the system, where for each experiment we prepare the system in the state $|\psi\rangle$ and then measure the spin of both particles for a particular choice of detector settings. The experimental results can be summarized in terms of a set of conditional probabilities $P(a x \wedge b y \mid A i \wedge B j)$ that give the probability that detectors $A, B$ register outcomes $x, y$ when the detectors are set to measure the spins along axes $\hat{e}_{i}, \hat{e}_{j}{ }^{4}$.

One can show that $|\psi\rangle$ is a rotationally symmetric state, and thus from symmetry considerations alone we can constrain the form of the conditional probabilities $P(a x \wedge b y \mid A i \wedge$ $B j$ ). We will formulate these symmetry constraints in terms of a postulate.

Postulate 1 (symmetry)

$$
\begin{aligned}
& P(a x \wedge b x \mid A i \wedge B j)=p\left(1-\delta_{i j}\right), \\
& P(a x \mid A i \wedge B j)=P(b x \mid A i \wedge B j)=1 / 2 .
\end{aligned}
$$

Physics does not depend on how we label the detector settings or the measurement outcomes.

The quantity $p$ that appears in equation (5) is a free parameter. The symmetry postulate says that the system remains physically unchanged if we permute detector settings $\{0,1,2\}$ or interchange measurement outcomes $u \leftrightarrow d$. Given the rotational symmetry of $|\psi\rangle$, it follows directly from physical considerations such as the isotropy of space and the conservation of angular momentum. Alternatively, we can view the system as a black box, in which the inputs are the detector settings and the outputs are the measurement outcomes, and from this perspective the symmetry postulate can be viewed as a purely empirical fact that summarizes the results of our experiments on the box.

Given that the system obeys the symmetry postulate, we can determine all of the conditional probabilities $P(a x \wedge b y \mid A i \wedge B j)$ from the single parameter $p$ (see table 1). To see this, note that

$$
P(a x \mid A i \wedge B j)=P(a x \wedge b x \mid A i \wedge B j)+P(a x \wedge b \bar{x} \mid A i \wedge B j),
$$

where we have defined $\bar{u}=d$ and $\bar{d}=u$. Substituting equations (5) and (6), we find that

$$
P(a x \wedge b \bar{x} \mid A i \wedge B j)=1 / 2-p\left(1-\delta_{i j}\right) .
$$

4 The wedge in ' $a x \wedge b y$ ' and ' $A i \wedge B j$ ' is meant to be read as 'and', so, for example, ' $a x \wedge b y$ ' indicates the situation in which detector $A$ registers outcome $x$ and detector $B$ registers outcome $y$. 
Table 1. Conditional probabilities $P(a x \wedge b y \mid A i \wedge B j)$ consistent with the symmetry postulate.

\begin{tabular}{lll}
\hline$P(a x \wedge b y \mid A i \wedge B j)$ & $x=y$ & $x \neq y$ \\
\hline$i=j$ & 0 & $1 / 2$ \\
$i \neq j$ & $p$ & $1 / 2-p$ \\
\hline
\end{tabular}

Table 2. Conditional probabilities $P(a x \wedge b y \mid A i \wedge B j)$ predicted by quantum mechanics.

\begin{tabular}{lll}
\hline$P(a x \wedge b y \mid A i \wedge B j)$ & $x=y$ & $x \neq y$ \\
\hline$i=j$ & 0 & $1 / 2$ \\
$i \neq j$ & $3 / 8$ & $1 / 8$ \\
\hline
\end{tabular}

Using the state vector $|\psi\rangle$ given in equation (3), it is straightforward to show that the value for $p$ predicted by quantum mechanics is $p=(1 / 2) \sin ^{2}(\pi / 3)=3 / 8$, and the conditional probabilities for this value of $p$ are shown in table 2 .

In what follows, we will investigate whether or not the conditional probabilities given in table 2 can be explained by a hidden variable theory. Such a theory consists of three components. First, we need a hidden variable, which describes some internal property of the system that cannot be directly measured, and a list of possible values that the hidden variable can assume. We will let $H q$ denote the situation in which the value of the hidden variable is $q$, and we will let $S i j q \equiv A i \wedge B j \wedge H q$ denote the situation in which detectors $A, B$ are set to measure the spins along axes $\hat{e}_{i}, \hat{e}_{j}$ and the value of the hidden variable is $q$. We will call $S i j q$ the state of the system. Next, we need a set of conditional probabilities $P(a x \wedge b y \mid$ Sijq $)$. These quantities give the probability that detectors $A, B$ register outcomes $x, y$ when the detectors are set to measure the spins along axes $\hat{e}_{i}, \hat{e}_{j}$ and the value of the hidden variable is $q$. We allow for the possibility that the measurement outcomes may only depend probabilistically on the hidden variable, so $P(a x \wedge b y \mid$ Sijq $)$ can lie anywhere in the range $[0,1]$. Finally, we need a set of probability distributions $P(H q \mid A i \wedge B j)$ for the hidden variable. These quantities give the probability that the hidden variable has value $q$ when detectors $A, B$ are set to measure the spins along axes $\hat{e}_{i}, \hat{e}_{j}$.

Once we have specified a hidden variable theory, we can determine its predictions for the conditional probabilities $P(a x \wedge b y \mid A i \wedge B j)$ as follows:

$$
P(a x \wedge b y \mid A i \wedge B j)=\sum_{q} P(a x \wedge b y \mid S i j q) P(H q \mid A i \wedge B j) .
$$

The hidden variable theory will agree with quantum mechanics if the conditional probabilities given by equation (9) agree with the conditional probabilities given in table 2. As we shall see, it is not difficult to construct hidden variable theories that agree with quantum mechanics. The problem is that these theories tend to violate additional constraints on what an acceptable theory should look like. In what follows, we will consider three specific constraints, each of which is formulated as a postulate that a hidden variable theory may or may not satisfy ${ }^{5}$ :

Postulate 2 (locality)

$$
P(\text { ax } \mid \text { Sijq })=P(a x \mid A i \wedge H q), \quad P(b x \mid \text { Sijq })=P(b x \mid B j \wedge H q) .
$$

For a given value of the hidden variable, the measurement outcome at one detector is independent of the setting of the other detector.

5 Similar sets of postulates are discussed in $[8,9]$. 
Postulate 3 (causality)

$$
P(a x \wedge b y \mid \text { Sijq })=P(a x \mid \text { Sijq }) P(b y \mid \text { Sijq }) .
$$

The hidden variable is the common cause of the measurement outcomes.

Postulate 4 (independence)

$$
P(H q \mid A i \wedge B j)=P(H q) .
$$

The value of the hidden variable is independent of the detector settings.

These postulates make explicit various assumptions that are implicit in the EPR argument; it is likely that EPR would have viewed each of these postulates as being necessary for an acceptable hidden variable theory. We will show that any theory that satisfies all three postulates cannot agree with quantum mechanics, but by violating any one of these postulates we can construct a theory that does agree with quantum mechanics. Note that whereas the symmetry postulate is experimentally testable, the locality, causality and independence postulates each involve the experimentally inaccessible hidden variable $q$, and thus cannot be directly tested. We can only learn about them indirectly, through the way they act together to constrain the conditional probabilities $P(a x \wedge b y \mid A i \wedge B j)$.

\section{Bell's theorem}

We begin by describing a simplified version of Bell's theorem: we show that any hidden variable theory that satisfies all four postulates cannot agree with quantum mechanics. To establish this result, we first show that for any theory that satisfies these postulates the measurement outcomes can be predicted with certainty from the system state Sijq. The argument proceeds as follows. The locality postulate states that

$$
P(a x \mid \text { Sijq })=P(a x \mid A i \wedge H q), \quad P(b x \mid \text { Sijq })=P(b x \mid B j \wedge H q) .
$$

For the special case $i=j$, these equations become

$$
P(a x \mid \text { Siiq })=P(a x \mid A i \wedge H q), \quad P(b x \mid S j j q)=P(b x \mid B j \wedge H q) .
$$

Thus, combining equations (13) and (14), we find that

$$
P(a x \mid \text { Sijq })=P(\text { ax } \mid \text { Siiq }), \quad P(b x \mid \text { Sijq })=P(b x \mid \text { Sjjq }) .
$$

Substituting equation (15) into the causality postulate, we obtain

$$
P(a x \wedge b y \mid S i j q)=P(a x \mid \text { Siiq }) P(b y \mid \text { Sjjq }) .
$$

We now want to show that $P(a x \mid S i i q), P(b y \mid S j j q)$ is either 0 or 1 . From the symmetry postulate, it follows that

$$
P(a x \wedge b x \mid A i \wedge B i)=0 .
$$

From equation (9), it follows that

$$
P(a x \wedge b x \mid A i \wedge B i)=\sum_{q} P(a x \wedge b x \mid S i i q) P(H q \mid A i \wedge B i) .
$$

Thus, from equations (17), (18) and the independence postulate, it follows that

$$
\sum_{q} P(a x \wedge b x \mid \operatorname{Siiq}) P(H q)=0 .
$$

Equation (19) tells us that for each value $q$, either $P(a x \wedge b x \mid$ Siiq $)$ or $P(H q)$ must be zero. But if $P(H q)=0$ then value $q$ never occurs, and can therefore be neglected. Thus, without 
loss of generality we can restrict our attention to the values $q$ for which $P(H q)>0$, and for this class of values

$$
P(a x \wedge b x \mid \text { Siiq })=0 .
$$

This equation says that for any value of the hidden variable, the measurement outcomes are opposite when the detector settings are the same. For the special case $x=y$ and $i=j$, the causality postulate states that

$$
P(a x \wedge b x \mid \text { Siiq })=P(a x \mid \text { Siiq }) P(b x \mid \text { Siiq }) .
$$

From equations (20) and (21), it follows that

$$
P(a x \mid \text { Siiq }) P(b x \mid \text { Siiq })=0 .
$$

By replacing $x$ with $\bar{x}$ in equation (22), we also have that

$$
P(a \bar{x} \mid \text { Siiq }) P(b \bar{x} \mid \text { Siiq })=0 .
$$

Note that

$$
P(a \bar{x} \mid \text { Siiq })=1-P(\text { ax } \mid \text { Siiq }), \quad P(b \bar{x} \mid \text { Siiq })=1-P(b x \mid \text { Siiq }) .
$$

From equations (23) and (24), it follows that

$$
(1-P(\text { ax } \mid \text { Siiq }))(1-P(b x \mid \text { Siiq }))=0 .
$$

From equations (22) and (25), it follows that

$$
P(\text { bx } \mid \text { Siiq })=1-P(\text { ax } \mid \text { Siiq }) .
$$

Substituting this result into equation (22), we find that

$$
P(\operatorname{ax} \mid \operatorname{Siqq})(1-P(\text { ax } \mid \text { Siiq }))=0
$$

Thus, $P($ ax $\mid$ Siiq $)$ is either 0 or 1 . Given a value $q$, define $q_{i}$ to be the outcome $x$ for which $P(\operatorname{ax} \mid$ Siiq $)=1$; that is, define $q_{i}$ such that

$$
P(\operatorname{ax} \mid \text { Siiq })= \begin{cases}1 & \text { if } x=q_{i}, \\ 0 & \text { otherwise. }\end{cases}
$$

Then equations (16) and (26) imply that

$$
P(a x \wedge b y \mid \text { Sijq })=\left\{\begin{array}{ll}
1 & \text { if } x=q_{i} \\
0 & \text { otherwise. }
\end{array} \text { and } \quad y=\bar{q}_{j},\right.
$$

Thus, when the system state is Sijq, we can predict with certainty that the measurement outcomes are $x=q_{i}$ and $y=\bar{q}_{j}$.

The above argument shows that for a given value $q$ the conditional probabilities $P(a x \wedge b y \mid$ Sijq $)$ only depend on the values of $q_{0}, q_{1}$ and $q_{2}$, each of which is either $u$ or $d$. Thus, there are only $2^{3}=8$ physically distinct values of the hidden variable. We will denote these values by $q_{0} q_{1} q_{2}$, so the set of physically distinct values for the hidden variable is $\{d d d, d d u, d u d, d u u, u d d, u d u, u u d, u u u\}$.

We have shown how the measurement outcomes can be determined from the system state; let us now see what can be said about the distribution of hidden variables. From the symmetry postulate, we find that

$$
p=P(a u \wedge b u \mid A 0 \wedge B 1)=P(a u \wedge b u \mid A 0 \wedge B 2) .
$$

From equation (9) and the independence postulate, we find that

$$
P(a u \wedge b u \mid A 0 \wedge B 1)=P(H u d d)+P(H u d u),
$$




$$
P(a u \wedge b u \mid A 0 \wedge B 2)=P(H u d d)+P(H u u d) .
$$

From equations (30)-(32), it follows that $P(H u d u)=P(H u u d)$. Similarly, we can show that

$$
\begin{aligned}
& P(H u u d)=P(H u d u)=P(H d u u), \\
& P(H d d u)=P(H d u d)=P(H u d d) .
\end{aligned}
$$

Thus, the distribution of hidden variables must have the form

$$
P(H q \mid A i \wedge B j)=P(H q)= \begin{cases}p_{0} & \text { for } q=d d d, \\ p_{1} & \text { for } q \in\{d d u, d u d, u d d\}, \\ p_{2} & \text { for } q \in\{u u d, u d u, d u u\}, \\ p_{3} & \text { for } q=\text { uиu }\end{cases}
$$

for some constants $p_{0}, p_{1}, p_{2}, p_{3}$. From equations (30), (31) and (35), it follows that $p=p_{1}+p_{2}$. But note that

$$
\sum_{q} P(H q \mid A i \wedge B j)=p_{0}+3 p_{1}+3 p_{2}+p_{3}=p_{0}+3 p+p_{3}=1
$$

so $p \leqslant 1 / 3$. Thus, a theory that satisfies all four postulates cannot give the value $p=3 / 8$ that is predicted by quantum mechanics, and therefore cannot agree with the quantum predictions for the conditional probabilities $P(a x \wedge b y \mid A i \wedge B j)$.

\section{Example hidden variable theory: causality postulate violated}

It is interesting to note that quantum mechanics itself can be viewed as a kind of degenerate hidden variable theory in which the hidden variable takes on a single value $q$, which can be thought of as the quantum state vector. The hidden variable theory corresponding to quantum mechanics is defined as follows. We stipulate that the conditional probabilities $P(a x \wedge b y \mid \operatorname{Sijq})$ are given by

$$
P(a x \wedge b y \mid \text { Sijq }) \equiv P(a x \wedge b y \mid A i \wedge B j),
$$

where $P(a x \wedge b y \mid A i \wedge B j)$ are the conditional probabilities predicted by quantum mechanics, as shown in table 2 . There is only one hidden variable $q$, so the distribution of hidden variables is given by

$$
P(H q \mid A i \wedge B j) \equiv 1 .
$$

Let us now check which postulates this theory satisfies and which it violates. Note that

$$
\begin{aligned}
& P(\text { ax } \mid \text { Sijq })=\sum_{y} P(a x \wedge b y \mid \text { Sijq })=1 / 2, \\
& P(b y \mid \text { Sijq })=\sum_{x} P(a x \wedge b y \mid \text { Sijq })=1 / 2 .
\end{aligned}
$$

Since $P($ ax $\mid$ Sijq $)$ and $P(b y \mid$ Sijq $)$ are independent of $i$ and $j$, the locality postulate is satisfied. The causality postulate is violated, since

$$
P(\operatorname{ax} \mid \text { Sijq }) P(b y \mid \text { Sijq })=1 / 4,
$$

but, for example, $P(a x \wedge b y \mid \operatorname{Sijq})=0$ for $i=j$ and $x=y$. The independence postulate is trivially satisfied, since there is only one possible value of the hidden variable:

$$
P(H q)=P(H q \mid A i \wedge B j)=1 .
$$


Table 3. Measurement outcomes $(x, y)$ given system state Sijq.

\begin{tabular}{lllll}
\hline$(x, y)$ & $q=u *$ & $q=u d$ & $q=d *$ & $q=d u$ \\
\hline$i=j$ & $(u, d)$ & $(u, d)$ & $(d, u)$ & $(d, u)$ \\
$i \neq j$ & $(u, u)$ & $(u, d)$ & $(d, d)$ & $(d, u)$ \\
\hline
\end{tabular}

By construction, the conditional probabilities $P(a x \wedge b y \mid A i \wedge B j)$ predicted by the theory agree with the predictions of quantum mechanics, so the theory satisfies the symmetry postulate. Note, however, that for this theory the measurement outcomes do not follow deterministically from the system state, and thus the theory does not succeed in restoring the common-sense view of the world. Of course, this lack of determinism is due to the fact that the theory is really just ordinary quantum mechanics transcribed into the language of hidden variable theories.

\section{Example hidden variable theory: locality postulate violated}

We will now show that by violating the locality postulate, we can construct a hidden variable theory that agrees with the predictions of quantum mechanics. The theory is defined as follows. There are four possible values for the hidden variable, which are denoted by $u *, u d, d *$ and $d u$. We stipulate that the measurement outcomes $(x, y)$ are deterministically related to the system state Sijq as shown in table 3, so, for example, if the system is in a state Sijq for which $i=j$ and $q=u *$, then

$$
P(a x \wedge b y \mid \text { Sijq })=\left\{\begin{array}{ll}
1 & \text { if } x=u \\
0 & \text { otherwise. }
\end{array} \quad \text { and } \quad y=d,\right.
$$

The probability distribution for the hidden variable is defined to be

$$
P(H q \mid A i \wedge B j) \equiv \begin{cases}3 / 8 & \text { for } \\ 1 / 8 & \text { for } \quad q \in\{u *, d *\},\end{cases}
$$

Let us now check which postulates this theory satisfies and which it violates. Since $P(H q \mid A i \wedge B j)$ does not depend on $i$ or $j$, the independence postulate is satisfied. For detector $A$, the locality postulate is satisfied. To see this, note that

$$
P(a u \mid \text { Sijq })=1-P(a d \mid \text { Sijq })= \begin{cases}1 & \text { if } q \in\{u *, u d\}, \\ 0 & \text { otherwise. }\end{cases}
$$

Since $P(a x \mid S i j q)$ is independent of $j$, detector $A$ satisfies the locality postulate. For detector $B$, however, the locality postulate is violated. For example, for $q=d *$,

$$
P(b u \mid \text { Sijq })=1-P(b d \mid \text { Sijq })= \begin{cases}1 & \text { if } i=j, \\ 0 & \text { otherwise. }\end{cases}
$$

Since $P(b x \mid$ Sijq $)$ depends on $i$, detector $B$ cannot satisfy the locality postulate. It is straightforward to check that this theory satisfies the symmetry postulate; from equation (9), it follows that the predicted value of $p$ is (take $i \neq j$ )

$p=P(a x \wedge b x \mid A i \wedge B j)=\sum_{q} P(a x \wedge b x \mid S i j q) P(H q \mid A i \wedge B j)=3 / 8$.

Thus, this theory agrees with the predictions of quantum mechanics. The problem with this theory is that it violates the locality postulate and thus involves the kind of action at a distance that EPR considered unacceptable. 
Table 4. Measurement outcomes $(x, y)$ given system state Sijq.

\begin{tabular}{lllll}
\hline$(x, y)$ & $q=u u$ & $q=u d$ & $q=d u$ & $q=d d$ \\
\hline$i=j$ & $(u, u)$ & $(u, d)$ & $(d, u)$ & $(d, d)$ \\
$i \neq j$ & $(u, u)$ & $(u, d)$ & $(d, u)$ & $(d, d)$ \\
\hline
\end{tabular}

Table 5. Hidden variable distribution given detector settings $A i \wedge B j$.

\begin{tabular}{lllll}
\hline$P(H q \mid A i \wedge B j)$ & $q=u u$ & $q=u d$ & $q=d u$ & $q=d d$ \\
\hline$i=j$ & 0 & $1 / 2$ & $1 / 2$ & 0 \\
$i \neq j$ & $3 / 8$ & $1 / 8$ & $1 / 8$ & $3 / 8$ \\
\hline
\end{tabular}

\section{Example hidden variable theory: independence postulate violated}

By violating the independence postulate, we can construct a different hidden variable theory that agrees with the predictions of quantum mechanics. This theory is defined as follows. There are four possible values for the hidden variable, which are denoted by $u u, u d, d u$ and $d d$. We stipulate that the measurement outcomes $(x, y)$ are deterministically related to the system state $\operatorname{Sijq}$ as shown in table 4, and the probability distribution $P(H q \mid A i \wedge B j)$ for the hidden variable is given by table 5 .

Let us now check which postulates this theory satisfies and which it violates. Since $P(H q \mid A i \wedge B j)$ depends on $i$ and $j$, the independence postulate is violated. Since $P(a x \wedge b y \mid S i j q)$ is independent of $i$ and $j$, the locality postulate is satisfied. It is straightforward to check that this theory satisfies the symmetry postulate; from equation (9), it follows that the predicted value for $p$ is (take $i \neq j$ )

$p=P(a x \wedge b x \mid A i \wedge B j)=\sum_{q} P(a x \wedge b x \mid S i j q) P(H q \mid A i \wedge B j)=3 / 8$.

Thus, this theory also agrees with the predictions of quantum mechanics. Unlike the previous theory, this theory is local. However, this theory has problems of its own: the value of the hidden variable depends on the detector settings. Since the hidden variable is fixed before the measurements occur, the theory involves a kind of advanced action in which the detector settings determine the prior value of the hidden variable. This type of hidden variable theory is usually ruled out on the grounds that such advanced action is physically implausible.

\section{Conclusion}

We have clarified the meaning and implications of Bell's theorem by showing how it applies to a simple model system, and by constructing three example hidden variable theories. The discussion should help illustrate two important points that are often misunderstood. First, Bell's theorem does not rule out all hidden variable theories; rather, it imposes a constraint on the types of hidden variable theories that can be constructed. Second, Bell's theorem does not even rule out local hidden variable theories unless it is supplemented with additional physical principles; as we have seen, it is logically possible to construct local hidden variable theories that do agree with quantum mechanics. We described one such local hidden variable theory in section 8 , and noted that this type of theory is usually ruled out on the grounds that it requires advanced action. 


\section{References}

[1] Einstein A, Podolsky B and Rosen N 1935 Phys. Rev. 47 777-80

[2] Bell J S 1964 Physics 1 195-200

[3] Laloe F 2001 Am. J. Phys. 69 655-701

[4] Dehlinger D and Mitchell M W 2002 Am. J. Phys. 70 903-10

[5] Mermin N D 1993 Rev. Mod. Phys. 65 803-15

[6] Bell J S 1966 Rev. Mod. Phys. 38 447-52

[7] Bohm D 1951 Quantum Theory (New York: Prentice-Hall) chapter 22

[8] van Fraassen B C 1982 Synthese 52 25-38

[9] Jarrett J 1989 Bell's theorem: a guide to the implications Philosophical Consequences of Quantum Theory ed J Cushing and E McMullin (Notre Dame: Univeristy of Notre Dame Press) 